(C) Facultad de Ciencias Biológicas UNMSM

\title{
Actividad leishmanicida de los extractos metanólicos de cuatro ecotipos de Lepidium peruvianum, Chacón (Brassicaceae)
}

\author{
Leishmanicidal activity of methanolic extract from four ecotypes of \\ Lepidium peruvianum, Chacón (Brassicaceae)
}

\author{
Libertad Alzamora', Hilda Solís², Marisol Rojas², Marisela Calderón ${ }^{2}$, Narda \\ Fajardo $^{2}$, Jenny Quispe ${ }^{1}$, Evelyn Alvarez ${ }^{1}$, Erasmo Colona ${ }^{1}$ y Dina Torres ${ }^{1}$
}

\begin{abstract}
${ }^{1}$ Universidad Nacional Mayor de San Marcos, Facultad de Ciencias Biológicas, Instituto de Investigaciones de Ciencias Biológicas Antonio Raimondi, Laboratorio de Inmunología.

Email Libertad Alzamora: lalzamorag@unmsm.edu.pe

2 Universidad Nacional Mayor de San Marcos. Facultad de Medicina Humana. Instituto de Medicina Tropical «Daniel Alcides Carrión».
\end{abstract}

\section{Resumen}

El tratamiento clásico de la leishmaniosis cutánea consiste en la inyección de 15-20 ampollas de Glucantine lo que ocasiona efectos secundarios, este hecho justifica la búsqueda de nuevos medicamentos motivando la presente investigación. El objetivo fue evaluar in vitro la actividad leishmanicida de los extractos metanólicos (EM) de los ecotipos blanco, rojo, morado y negro de Lepidium peruvianum, Chacón (también conocida como Lepidium meyenii Walp.), sobre el crecimiento de Leishmania braziliensis peruviana. Los promastigotes alcanzaron la fase de crecimiento exponencial al quinto día de cultivo a $27^{\circ} \mathrm{C}$ en el medio bifásico Columbia, suplementado con $15 \%$ de sangre desfibrinada de carnero, en ese momento se enfrentaron, por separado, con los EM a concentraciones de 50, 100, 200 y $400 \mu \mathrm{g} / \mathrm{ml}$. Los recuentos se hicieron diariamente con cámara Neubauer. La máxima disminución de promastigotes se produjo al segundo día de enfrentamiento para el ecotipo morado $(17,41 \%$ de viabilidad) empleando 400 $\mu \mathrm{g} / \mathrm{ml}$. El efecto leishmanicida estaría relacionado con los alcaloides imidazólicos presentes en el EM. Se concluye que al segundo día de enfrentamiento con el EM, el ecotipo morado presenta la mayor actividad leishmanicida seguido del ecotipo blanco.

Palabras clave: Leishmania braziliensis peruviana, Lepidium peruvianum; Lepidium meyenii, actividad leishmanicida, plantas medicinales peruanas, ecotipos de Maca.

\section{Abstract}

The classic treatment of the cutaneous leishmaniosis consists on the injection of 15-20 ampoule of Glucantine what causes serious secondary effects. This fact justifies the search of new medications what motivated the present investigation. The objective was to evaluate the leishmanicidal activity of the methanolic extracts (ME) of the white, red, purple and black ecotypes of Lepidium peruvianum Chacón (at present Lepidium meyenii Walp.) about the growth of Leishmania braziliensis peruviana in vitro. The promastigotes reached the logarithmic phase to the fifth day of cultivation at $27^{\circ} \mathrm{C}$ in the two-phase Columbia medium with $15 \%$ of defibrinated sheep blood and they faced, for separate, with the ME to concentrations of 50,100, 200 and 400 $\mu \mathrm{g} / \mathrm{ml}$. The recounts were made daily with camera Neubauer. The maximum decrease of promastigotes ( $17.41 \%$ of viability) took place to the second day for the purple ecotype with the concentration of $400 \mu \mathrm{g} / \mathrm{ml}$. The leishmanicidal effect would be related with the imidazolic alkaloids, glucosinolates, flavonoids, tannins and saponines present in the ME. The conclusion is that only $\mathrm{ME}$ of the white and purple ecotypes presents leishmanicidal activity, at second day of culture.

Keywords: Leishmania braziliensis peruviana, Lepidium peruvianum, Lepidium meyenii, Leishmanicidal activity, Methanolic extract, Peruvian Medicinal plants, ecotype of Maca.

\section{Introducción}

La leishmaniosis es una enfermedad crónica, de baja patogenicidad, alta morbilidad, metaxénica y zoonótica, producida por protozoarios hemoflagelados del género Leishmania, (Sarcomastigophora, Kinetoplastida). Esta enfermedad afecta a 12 millones de personas alrededor del mundo, estimándose 2 millones de nuevos casos por año (OMS, 2002); es transmitida por dípteros hematófagos del género Lutromyia en el Nuevo Mundo y Pblebotomus en el Viejo Mundo; tiene como reservorio

Nota del Editor: El nombre Lepidium peruvianum Chacón debe ser considerado sinónimo de Lepidium meyenii Walp. (ver Germplasm Resources Information Network, United States Department of Agriculture, Agricultural Research Service, en http:/ /www.ars-grin.gov/cgi-bin/npgs/html/taxon.pl?21767, acceso 08/02/07) preferencias del autor obligan a mantener L. peruvianum Chacón en el texto. a animales vertebrados como roedores y perros; su ciclo de vida es digenético e incluye una forma promastigote extracelular en el vector y el estado amastigote no flagelado intracelular dentro de fagocitos mononucleares de hospederos vertebrados (Alexander et al., 1999; OGE-INS Lima-Perú, 2000).

Según la especie de Leishmania y dependiendo de la respuesta inmune del hospedero la enfermedad compromete la piel, mucosas y vísceras (Lucas et al., 1998).

En el Perú, la leishmaniosis es la segunda endemia de tipo tropical y la tercera causa de morbilidad por enfermedades transmisibles luego de la malaria y la tuberculosis (Neyra et al., 1997), y se presenta en dos formas: el $75 \%$ de los casos reportados corresponden a la forma cutáneo-andina o «uta» (L. braziliensis peruviana) y el $25 \%$ a la forma cutáneo mucosa (L. braziliensis 
braziliensis) (Lucas et al., 1998). El tratamiento consiste en la administración de antimoniales pentavalentes pero su administración causa efectos secundarios como mialgia, pancreatitis, insuficiencia renal, neuropatía periférica, hepatotoxicidad, y cardiotoxicidad (Croft y Yardley, 2002). En la actualidad existen varias drogas que han sido ensayadas para el tratamiento quimioterápico de Leishmaniosis cutánea pero muchas de ellas son nuevas formulaciones de drogas diseñadas hace 50 años; además se ha presentado resistencia a las drogas clásicamente empleadas, lo cual hace necesaria la búsqueda de nuevos medicamentos que reemplacen o complementen el tratamiento actual (Croft y Yardley, 2002; Osorio et al., 2005).

Los extractos de plantas medicinales o componentes derivados de éstas son una fuente valiosa para el tratamiento de ésta y otras enfermedades (Carvalho y Ferreira, 2001).

La maca, Lepidium peruvianum Chacón (también conocida como Lepidium meyenii Walp.) es la única Brassicaceae domesticada de los andes peruanos (3700 - $4500 \mathrm{~m}$ de altitud). La raíz es usada para el consumo humano por su gran valor nutricional desde tiempos precolombinos; además contiene metabolitos secundarios que le confieren propiedades medicinales tales como la estimulación en la producción de folículos ováricos en ratas (Chacón, 1961); efecto sobre la espermatogénesis de ratas (Gonzales et al., 2001) esta propiedad podría deberse a la presencia de isotiocianatos biológicamente activos: benzil-isotiocianato y p-metoxibenzil isotiocianato (Li et al., 2001), además se le atribuye efecto antioxidante (Sandoval et al., 2002).

Entre los componentes fitoquímicos destacan ácidos grasos esenciales como el linolénico y el oleico, flavonoides y saponinas (Dini et al., 1994). Otros compuestos importantes son los esteroles, alcaloides (Cui et al., 2003), derivados benzilados (Muhammad et al., 2002) y glucosinolatos (Ganzera et al., 2002).

Aunque no se han encontrado referencias respecto al efecto leishmanicida de L. peruvianum; la presencia en esta especie de alcaloides imidazólicos, benzil glucosinolatos, taninos, flavonoides y saponinas, todos ellos compuestos muy activos frente a Leishmania y a trofozoitos de Giardia intestinalis (Echevarría y Torres, 2001), podrían causar un efecto leishmanicida. Por esta razón se planteó como objetivo evaluar la posible actividad leishmanicida del extracto metanólico preparado a partir de maca pulverizada de cuatro ecotipos: blanco, rojo, morado y negro.

\section{Material y métodos \\ Ecotipos de Maca}

Se utilizaron cuatro ecotipos (blanco, morado, negro y rojo) de L. peruvianum Chacón (Brassicaceae) comúnmente conocida como «Maca», procedentes del Valle de Pampas, Provincia de Tayacaja, Departamento de Huancavelica.

\section{Preparación del Extracto}

Se preparó un extracto metanólico (EM) de cada uno de los ecotipos, utilizando maca pulverizada obtenida a partir de las raíces y macerada durante 10 días en metanol QP (1:2). El macerado se filtró y se concentró en estufa de aire circulante a $40^{\circ} \mathrm{C}$ hasta obtener $1 / 4$ del volumen inicial, luego se secó en estufa a $40^{\circ} \mathrm{C}$, hasta evaporación total del solvente. A partir de éste se prepararon concentraciones de 50,100, 200 y $400 \mu \mathrm{g} / \mathrm{ml}$ las mismas que se enfrentaron a los cultivos en fase de crecimiento exponencial (fase log) de $L$.
Tabla 1. Promedios de actividad leishmanicida por dia del experimento. Se muestran los resultados de la actividad del extracto metanólico de las raíces de cuatro ecotipos de Lepidium peruvianum, Maca, para la concentración del extracto metanólico de $100 \mu \mathrm{g} / \mathrm{ml}$.

\begin{tabular}{lccccc}
\hline \multicolumn{7}{c}{ Días del cultivo } \\
Ecotipo & $\mathbf{1}$ & $\mathbf{2}$ & $\mathbf{3}$ & $\mathbf{4}$ & $\mathbf{5}$ \\
\hline Blanco & 4240 & 6765 & 1300 & 620 & 2380 \\
Morado & 5020 & 3780 & 3250 & 3320 & 4310 \\
Negro & 3430 & 2350 & 4450 & 6040 & 2810 \\
Rojo & 1770 & 2280 & 2780 & 3320 & 3740 \\
Control & 2610 & 3730 & 5210 & 5410 & 4030 \\
\hline
\end{tabular}

braziliensis peruviana, se empleó solución salina como diluyente.

\section{Cultivo del Parásito}

Los promastigotes de L. braziliensis perwviana fueron cultivados a $27^{\circ} \mathrm{C}$ en medio bifásico Columbia $\left(\mathrm{BBL}^{\mathrm{TM}}\right)$ suplementado con $15 \%$ de glóbulos rojos de carnero (GRC), se hicieron recuentos diarios a fin de determinar la fase logarítmica del parásito. El medio comprende una fase líquida (solución salina), como antimicrobiano se empleó Amikacina.

\section{Actividad leishmanicida}

Para el ensayo in vitro se utilizaron cultivos de promastigotes de $L$. braziliensis peruviana en fase log. Luego de 5 días de cultivo a la fase líquida se le añadió el extracto metanólico de cada ecotipo de L. peruvianum (blanco, rojo, morado y negro). Se emplearon concentraciones de 50, 100, 200 y $400 \mu \mathrm{g} / \mathrm{ml}$ de EM; los ensayos se realizaron por triplicado para cada ecotipo, considerando como controles cultivos sin EM.

El recuento de parásitos se realizó diariamente desde el primero hasta el quinto día de incubación con el EM. Para el recuento del parásito se empleó cámara de Neubauer (400x) lo que además permitió evaluar la morfología y la supervivencia del parásito, esta última, relacionada con su movimiento. La actividad leishmanicida (reducción en la cantidad de promastigotes) se determinó aplicando la siguiente fórmula:

\section{Actividad leishmanicida $=[100-(L 2 / L 1) \times 100]$}

Donde L1 es el número de células viables en el cultivo control y L2 es el número de células viables después del tratamiento con los extractos. El porcentaje de viabilidad se determinó por comparación con el número de promastigotes de los controles. Los resultados se analizaron mediante la prueba t de Student.

\section{Resultados}

En general, a la concentración de $100 \mu \mathrm{g} / \mathrm{ml}$ el ecotipo morado tuvo mejor efecto que el ecotipo rojo $(p=0,04)$, pero respecto a los demás ecotipos no se observó diferencia significativa.

A la concentración de $400 \mu \mathrm{g} / \mathrm{ml}$, el ecotipo morado fue más efectivo que los demás ( $p>0,05)$. Asimismo, a la concentración de 50 y $200 \mu \mathrm{g} / \mathrm{ml}$ no se evidenció diferencia significativa entre los 4 ecotipos.

El análisis de los datos de acuerdo a los días de cultivo, permitió determinar que la dosis de $400 \mu \mathrm{l}$ de EM del ecotipo morado de L. peruvianum mostró mejor actividad leishmanicida (82,58\%) reduciendo la viabilidad de los promastigotes a 17,41\% el segundo día de cultivo, esta actividad disminuyó al tercero, cuarto y quinto día de cultivo) (Tabla 3, Fig. 1). 


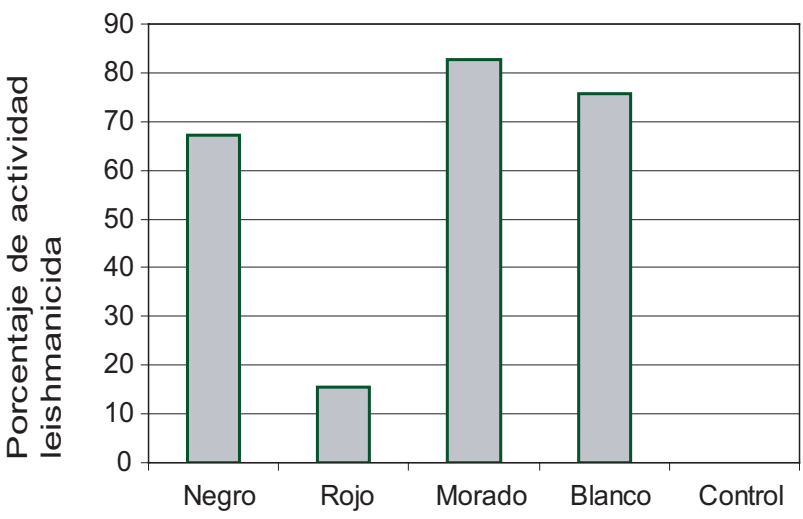

Figura 1. Efecto del extracto metanólico (EM) de cuatro ecotipos de Lepidium peruvianum, Maca, sobre cultivos de Leishmania braziliensis peruviana, a los dos días de cultivo. La máxima actividad leishmanicida $(82,58 \%)$ fue observada al segundo día, con la dosis de $400 \mu \mathrm{g} / \mathrm{ml}$ de EM para el ecotipo morado de maca, (porcentaje de viabilidad: $17,41 \%)$. Al tercer, cuarto y quinto día de cultivo la viabilidad se redujo a $19,15,29,86$ y $48,78 \%$, respectivamente.

El EM del ecotipo blanco, provocó la disminución del número de promastigotes al segundo día de cultivo; sin embargo, para este ecotipo se evidenció un notorio incremento en el número de promastigotes al tercer día, el mismo que en los días subsiguientes disminuyó (Tabla 3).

Los EM de los ecotipos negro y rojo de L. peruvianum no mostraron actividad leishmanicida significativa. Al quinto día, todos los cultivos presentaron igual número de promastigotes que el control (Tablas 1, 2 y 3).

La actividad leishmanicida se corroboró por la manifestación de alteraciones morfológicas y por la presencia de células lisadas en los cultivos con EM.

\section{Discusión}

La actividad leishmanicida de los alcaloides ha sido demostrada por varios investigadores (Chan et al., 2001). Por ejemplo Tempone et al. (2005) reportaron la actividad in vitro de alcaloides isoquinolínicos contra promastigotes de Leishmania chagasi, los extractos con alcaloides fueron obtenidos de Annona coriacea, Annona crassiflora, entre otras plantas. Respecto a la maca, esta crucífera contiene alcaloides imidazólicos que están presentes en los extractos metanólicos empleados (Dini et al., 1994; Cui et al., 2003; Ganzera et al., 2002).

Tabla 2. Promedios de actividad leishmanicida por día del experimento. Se muestran los resultados de la actividad del extracto metanólico de las raíces de cuatro ecotipos de Lepidium peruvianum, Maca, para la concentración del extracto metanólico de $200 \mu \mathrm{g} / \mathrm{ml}$

\begin{tabular}{lccccc}
\hline & \multicolumn{5}{c}{ Días de cultivo } \\
Ecotipo & 1 & 2 & 3 & 4 & 5 \\
\hline Blanco & 9110 & 6765 & 1300 & 620 & 4690 \\
Morado & 7460 & 6520 & 4260 & 4440 & 6310 \\
Negro & 4420 & 4730 & 6620 & 12040 & 5870 \\
Rojo & 2330 & 3190 & 5010 & 2710 & 5210 \\
Control & 9750 & 3790 & 4230 & 4490 & 5370 \\
\hline
\end{tabular}

Tabla 3. Promedios de actividad leishmanicida por día del experimento. Se muestran los resultados de la actividad del extracto metanólico de las raíces de cuatro ecotipos de Lepidium peruvianum, Maca, para la concentración del extracto metanólico de $400 \mu \mathrm{g} / \mathrm{ml}$.

\begin{tabular}{lccccc}
\hline & \multicolumn{5}{c}{ Días de cultivo } \\
Ecotipos & $\mathbf{1}$ & $\mathbf{2}$ & $\mathbf{3}$ & $\mathbf{4}$ & $\mathbf{5}$ \\
\hline Blanco & 6540 & 920 & 13590 & 4490 & 3360 \\
Morado & 4580 & 660 & 810 & 1490 & 2620 \\
Negro & 2100 & 1220 & 3690 & 5740 & 5230 \\
Rojo & 4320 & 1880 & 2720 & 1710 & 5570 \\
Control & 9750 & 3790 & 4230 & 4490 & 5370 \\
\hline
\end{tabular}

Otros metabolitos secundarios también ejercen actividad contra Leishmania, así por ejemplo las saponinas triterpenoides obtenidas de Careya arborea atacan a Leishmania donovani (Mandal et al., 2005). Torres-Santos et al. (2004) demostraron la actividad leishmanicida de los triterpenoides de Pourouma guianensis. La maca (ecotipo amarillo) también presenta estos metabolitos (Alzamora, 2003). Por los resultados obtenidos, se puede deducir que las concentraciones de alcaloides imidazólicos, benzil glucosinolatos, taninos, flavonoides y saponinas contenidos en L. peruvianum difieren entre ecotipos, y serían las causas de las diferencias en el efecto leishmanicida. El elevado incremento de promastigotes a las $72 \mathrm{~h}$ de cultivo, empleando $400 \mu \mathrm{g}$ de EM del ecotipo blanco, podría estar relacionado con la presencia de micronutrientes; Monteghirfo (2006), determinó que el ecotipo blanco presentaba mejor calidad nutritiva (46,7\% de aminoácidos esenciales) que el ecotipo amarillo (33,2\%); de igual forma el ecotipo morado también tiene un importante contenido de aminoácidos esenciales $(44,1 \%)$. Es posible que estos micronutrientes beneficien el crecimiento de los promastigotes, siendo un limitante para el uso de maca de ecotipo amarillo en personas que padecen de leishmaniosis.

El extracto metanólico del ecotipo morado se presenta como una promisoria posibilidad que debe continuar estudiándose, ya que afecta a los promastigotes de Leishmania braziliensis peruviana, por lo que estos resultados se constituyen en indicadores de la susceptibilidad del estadio de amastigote que se desarrolla en el hospedero vertebrado.

\section{Agradecimientos}

Los autores agradecen el financiamiento otorgado por el Consejo Superior de Investigaciones-UNMSM, Proyecto $\mathrm{N}^{\circ}$ 051001061.

\section{Literatura citada}

Alexander J., A.R. Satoskar \& D.G. Rusell. 1999. Leishmania species: Models of intracellular parasitism. Journal of Cell Science 112: 2993-3002.

Carvalho P. B. y E. Ferreira. 2001. Leishmaniasis phytotherapy. Nature's leadership against an ancient disease. Fitoterapia 72(6): 599-618.

Chacón G. 1961. Estudio fitoquímico de Lepidium meyenii Walp. Tesis de Bachiller en Ciencias Biológicas. Facultad de Ciencias, Universidad Nacional Mayor de San Marcos. Lima. Pp. 46.

Chan-Bacab M.J. \& L.M. Peña-Rodríguez. 2001. Plant natural products with leishmanicidal activity. Nat. Prod. Rep 18: 674-688.

Croft S.L. \& V. Yardley. 2002. Chemotherapy of Leishmanisis. Current Pharmacology 8: 319-342. 
Cui B., B. Zheng, K. He \& Q. Zheng. 2003. Imidazole alkaloids from Lepidium meyenii. J. Nat. Prod. 66: 1101-1103.

Dini A., G. Migliuolo, L. Rastrelli, P. Saturnino y O. Schettino. 1994. Chemical composition of Lepidium meyenii. Food Chemistry 49: 347-349.

Echevarría A. \& D. Torres. 2001. Efecto de un extracto de Petiveria alliacea Lin sobre el crecimiento de Giardia lamblia in vitro. Rev. Cubana Med. Milit. 30:161-165.

Ganzera M., J. Zhao, I. Muhammad \& I. Khan. 2002. Chemical profiling and standardization of Lepidium meyenii (Maca) by reversed phase high performance liquid chromatography. Chem. Pharm. Bull. 50(7):988-91.

Gonzales G.F., A. Ruiz, C. Gonzales, L. Villegas \& A. Córdova. 2001. Effect of Lepidium meyenii (Maca) roots on spermatogenesis of male rats. Asian J. Androl 3:231-233.

Li G., Ammermann U. y C.F. Quiroz. 2001. Glucosinolate contents in Maca (Lepidium peruvianum Chacón), seeds, sprouts; nature plants and several derived commercial products. Economy Botany 55:255-262.

Lucas C., A. Franke, M. Cachay, A. Tejada, M. Cruz, R. Kreutzer, D. Barrer, S. McCann \& D. Watts. 1998. Geographic distribution and clinical description of leishmaniasis cases in Peru. American Journal of Tropical Medicine and Hygiene 59:312-17.

Manda, D., N. Panda, S. Kumar, S. Banerjee, N. Mandal \& N. Sahu. 2006. A triterpenoid saponin possessing antileishmanial activity from the leaves of Careya arborea. Phytochemistry 67: $183-190$.
Monteghirfo, M. 2006. Caracterización bioquímica de las proteínas de las raíces de tres ecotipos de maca (Lepidium peruvianum G. Chacón) procedentes de Junín. Tesis para optar el Título Profesional de Biólogo con Mención en Genética. Facultad de Ciencias Biologicas, Universidad Nacional Mayor de San Marcos, Lima, Perú. 42 Págs.

Muhammad I., D. Zhao \& I. Khan. 2002. Constituents of Lepidium meyenii «maca». Phytochemistry 59: 105-110.

Neyra D. 1997. Las Leishmaniasis en el Perú. Folia Dermatológica Peruana 8:51-55.

OGE-INS. 2000. Módulos Técnicos Serie Documentos Monográficos $N^{\circ} 8$. INS, Lima, Perú.

OMS. 2002. Control of the leishmaniasis. Geneva, World Health Organization Expert Comite. Technical Report of Series No793.

Osorio E.J., S.M. Robiedo, G.S. Arango y C. Muskus. 2005.

Sandoval M., N. Okuhama, F. Angles, V. Melchor, L. Condezo, Lao J. \& J. Millar. 2002. Antioxidant activity of the cruciferous vegetable Maca (Lepidium peruvianum). Food Chemistry 79: 207-213

Tempone A.G., S.E. Treiger, H.F. Andrade, N.C. Amorin, A. Yogi, C. Salerno, D. Bachiega, F. N. Lupo, S.V. Bonotto \& D.C.H. Fischer. 2005. Antiprotozoal activity of Brazilian plants extract from isoquinoline alkaloid-producing families. Photochemistry 12:382-390.

Torres-Santos, E., D. Lopes, R. Rodrígues, J. Carauta, C. Bandeira, M. Kaplan \& B. Rossi-Bergman. 2004. Antileishmanial activity of isolated triterpenoids from Pouroma guianensis. Phytomedicine 11: 114-120. 